programmes qui conviennent aux partenaires. Des précédents, concernant les recherches sur les rétrovirus ou sur les agents transmissibles non conventionnels, sont là pour rappeler combien il est difficile d'anticiper le futur, et combien la diversité thématique est une force: on ne peut opposer la recherche heuristique à la recherche finalisée.

La question fondamentale est de savoir quelles sont la part et la recevabilité des arguments économiques dans le développement actuel des théories élitistes de la recherche. Pour maintenir une recherche combinatoire, le problème est celui du faible nombre de postes ouverts au concours chercheur. Les chercheurs demandent des moyens pour leur recherche, en financement et en personnels. Ils sont conscients que les difficultés économiques se répercutent sur le financement de la recherche publique. Mais pas sur leurs convictions

\section{RÉFÉRENCE}

1. Desplan C. Quand «rentrer» ne signifie pas uniquement «au Cnrs». Med Sci 1997 13: 213-5.

\section{Gabrielle Planelles}

Directeur de recherche, Inserm U.467, Faculté de Médecine, hôpital NeckerEnfants Malades, 156, rue de Vaugirard, 75730 Paris Cedex 15, France.

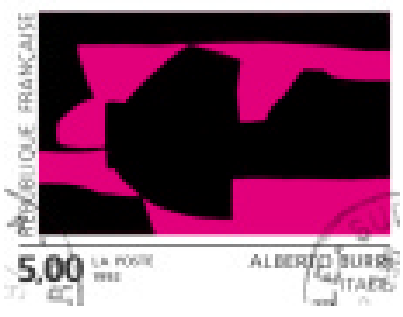

\title{
Contribution au débat sur le diagnostic préimplantatoire
}

Monsieur

J'ai lu avec grand intérêt les articles de P. Jouannet [1], J. Testard et B. Sèle [2]. La question de l'eugénisme est une des plus sensibles et suscite volontiers des réactions émotives. J'aimerais que, plutôt que de produire des arguments nés de l'émotion, les faits soient considérés objectivement, et cela dans un texte court et non dans une dissection longue et complexe de chaque terme utilisé.

A propos du débat sur eugénisme et diagnostic préimplantatoire, j'aimerais souligner que la législation française réprime spécifiquement toute pratique eugéniste. D'après ce que j'ai cru comprendre, il s'agit de la sélection organisée de caractères ou de gènes, ce qui implique une entreprise à grande échelle et non pas le soulagement des souffrances prévues d'un être futur à l'échelon singulier de la famille. A ce niveau, les décisions sont prises au cas par cas. Le diagnostic préimplantatoire s'est

blèmes de couples individuels et non pour mettre en œuvre des pratiques fantasmatiques d'inspiration raciste sur le modèle nazi, ou d'autres rappelant les pratiques eugénistes concernant les stérilisations forcées en vue d'empêcher la procréation de certains handicapés.

En outre, au Royaume-Uni, par exemple, existent les procédures d'agrément prévues par la loi qui servent de "garde-fou» contre les extrêmes, système qui peut toujours être complété lorsque change le contexte ; la Human fertilisation and embryology authority étudie la question de plus près en ce moment même par le biais d'un groupe de travail spécialement formé à cet effet. De même, en France, les décrets d'application devront permettre de délimiter précisément les évolutions utiles de ce champ d'application. La révision de la loi française prévue en 1999 interviendra justement à un moment idéal pour préciser ces extensions; cela permet d'en commencer dès aujourd'hui une analyse précise et réfléchie de manière à anticiper les accusations injustifiées contre une technique qui, impliquant la fécondation in vitro, est si lourde pour un couple que le spectre de son application en routine pour sélectionner des caractéristiques insignifiantes pour un futur enfant n'est guère envisageable

\section{RÉFÉRENCES}

1. Jouannet P. Peut-on intégrer l'embryon humain dans le champ médical et scientifique. Med Sci 1996; 12: 1331-3.

2. Testard J, Sèle B. Le diagnostic préimplantatoire n'est pas un diagnostic prénatal précoce. Med Sci 1996; 12: 1398-401.

\section{Françoise Shenfield}

Maître de conférence de clinique gynécologique et obstétricale, maître en Droit médical et Éthique, Fertility Unit, Middx Hospital and University College Medical School, Mortimer Street, London W1A 8AA, Royaume-Uni. 